\title{
Minimal scarring from giant intramuscular lipoma excision with the harmonic scalpel
}

\section{Jun Hyeok Kim, Yeon Ji Lee, Deuk Young $\mathrm{Oh}$}

Department of Plastic and Reconstructive Surgery, College of Medicine, The Catholic University of Korea, Seoul, Korea
Lipoma is the most frequent benign mesenchymal tumor. Giant intramuscular lipomas, defined by a measurement greater than $10 \mathrm{~cm}$ in any dimension, reside deep to the fascia and originate within the muscle layer. Traditionally, the length of the incision required for excision is equal to or greater than the diameter of the giant intramuscular lipoma. However, because of injury to the surrounding tissues from excessive traction and thermal injury from the energy delivered by the device, long and noticeable scars with a hypertrophic tendency develop, resulting in patient dissatisfaction. Although some esthetically-favorable methods have been reported, including liposuction and liposuction-assisted excision, these methods did not sufficiently excise lipomas, and led to a higher recurrence rate. In contrast, the ultrasonic harmonic scalpel can excise large and deep-seated lipomas with minimal scarring. The ultrasonic harmonic scalpel requires a shorter incision, and it provides a sufficient view of the surgical field, thereby avoiding unnecessary traction applied to the skin and reducing surrounding tissue injury trauma, which results in hypertrophic scars.

Keywords Cicatrix / Lipoma / Ultrasonics

\section{INTRODUCTION}

Lipoma is a benign mesenchymal tumor and the most common soft tissue tumor [1]. Giant intramuscular lipomas are deep-seated within the muscle [2], and are defined by a measurement greater than $10 \mathrm{~cm}$ in any dimension [3]. Most patients with a giant lipoma desire surgical excision for esthetic reasons, and others want surgical resection because of pain secondary to compression or expansion of an adjacent peripheral nerve [4,5].

Traditionally, a long surgical incision has been necessary for total excision of a deep and large intramuscular lipoma, resulting in a long scar and esthetic dissatisfaction. Reducing the scar length by

Received: Apr 23, 2019 Revised: May 11, 2019 Accepted: May 13, 2019 Correspondence: Deuk Young Oh Department of Plastic Surgery, Seoul St. Mary's Hospital, College of Medicine, The Catholic University of Korea, 222 Banpo-daero, Seocho-gu, Seoul 06591, Korea

Tel: +82-2-2258-6143, Fax: +82-2-594-7230, E-mail: ohdeuk1234@hanmail.net

Copyright @ 2019 The Korean Society for Aesthetic Plastic Surgery.

This is an Open Access article distributed under the terms of the Creative Commons Attribution Non-Commercial License (http://creativecommons.org/licenses/by-nc/4.0/) which permits unrestricted non-commercial use, distribution, and reproduction in any medium, provided the original work is properly cited. www.e-aaps.org shortening the incision results in tissue injury around the incision margin from traction, which may hinder healing and lead to the formation of hypertrophic scars [6,7].

Some other methods have been suggested to shorten the scar, including suction-assisted surgical excision, endoscopic-assisted liposuction, and endoscopic excision for removing moderate and large lipomas [8-10]. However, it is difficult to achieve complete removal of a lipoma and its entire capsule with these methods, and their recurrence rate has been reported to be high [11].

The present study suggests that using an ultrasonic energy device minimizes the scar after excision of a giant intramuscular lipoma.

\section{CASE REPORT}

A 62-year-old male patient was admitted with a giant intramuscular lipoma located in his anterolateral abdominal wall (Fig. 1). The patient desired complete removal of the lipoma for diagnostic and cosmetic purposes, but he was concerned about postoperative scarring because he had the tendency to form hypertrophic scars, as seen on his right chest (Fig. 1). Computed tomography (CT) revealed a lipoma measuring $10 \times 10 \mathrm{~cm}$ located deep in the external oblique muscle (Fig. 2). Although a benign lipoma was suspected, 


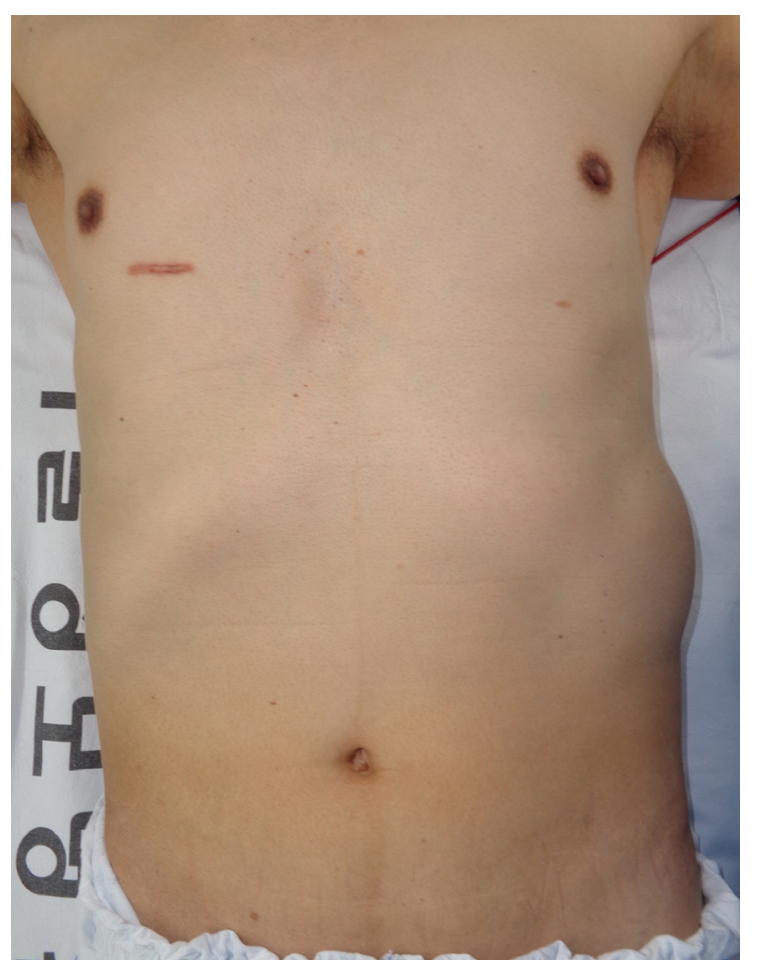

Fig. 1. A preoperative clinical photo showing a huge mass on the left anterolateral abdominal wall and a previous hypertrophic scar on the patient's right chest.

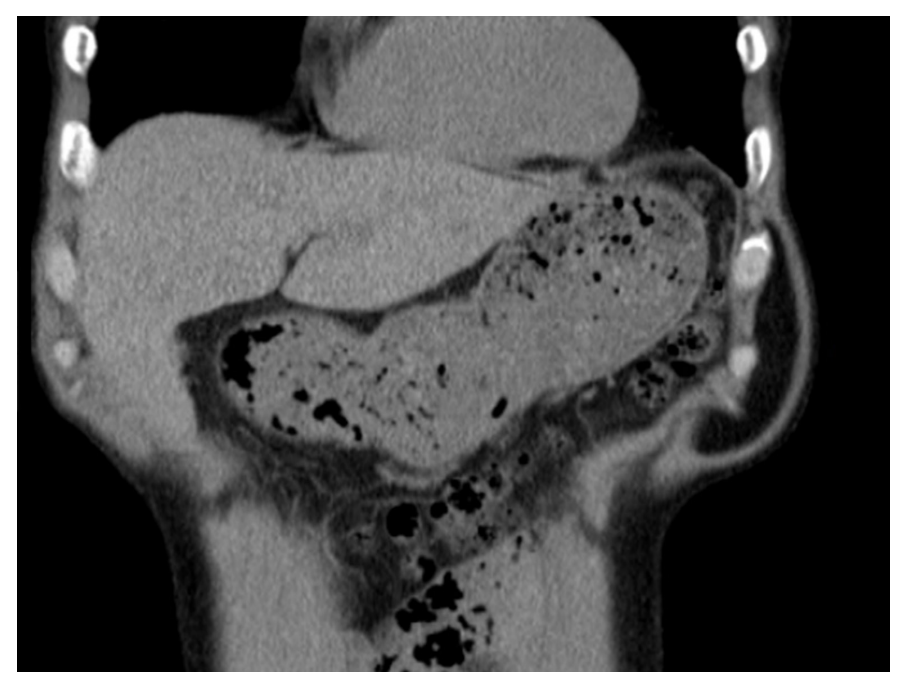

Fig. 2. Computed tomography revealed a lipoma measuring $10 \times 10 \mathrm{~cm}$ located deep in the external oblique muscle.

CT did not completely exclude the possibility of liposarcoma.

With the patient under general endotracheal anesthesia, surgical excision was performed. The skin incision was only $3.5 \mathrm{~cm}$ long. After dissection of the subcutaneous layer, the muscle was split to expose the lipoma (Fig. 3A). A mass, seated under the muscle layer, was completely removed using a harmonic scalpel (Harmonic HD;
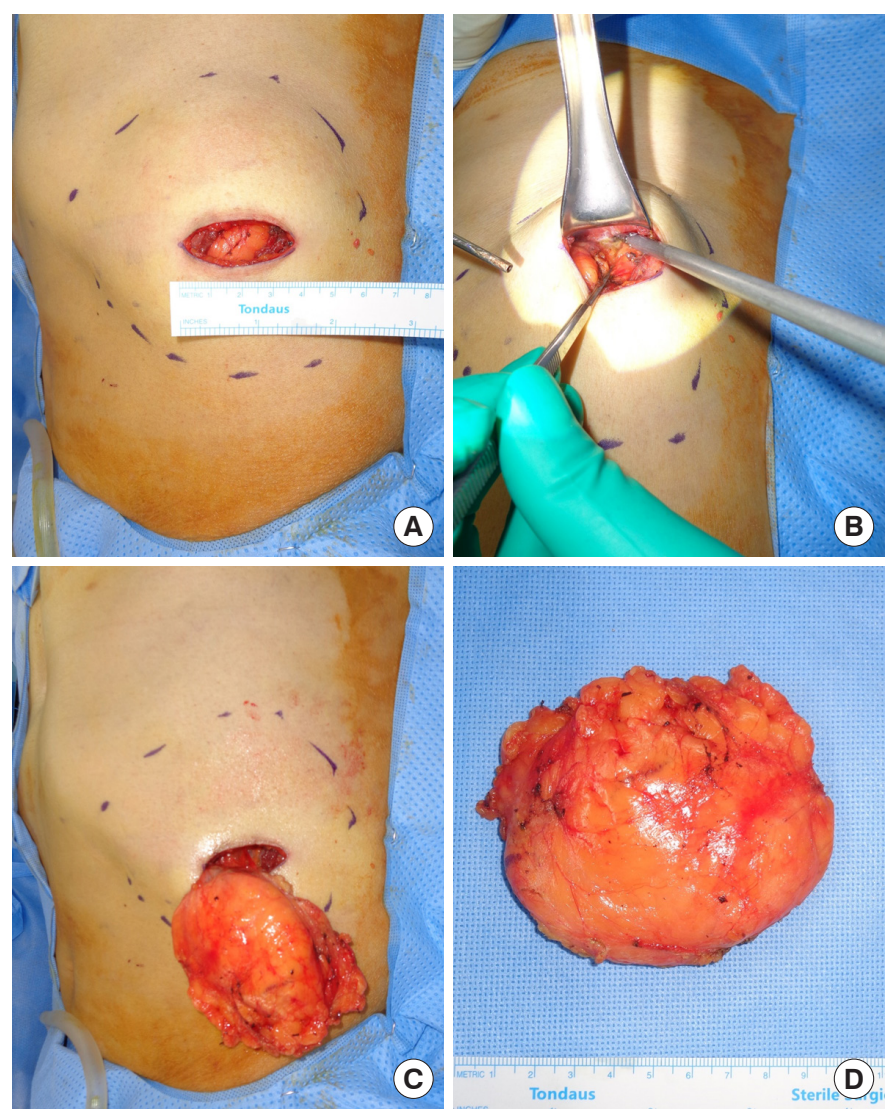

Fig. 3. Compared to the size of the lipoma, a relatively-short 3.5-cmsized skin incision was made (A) for dissection of the intramuscular lipoma with the Harmonic scalpel. (B) Dissected lipoma (C) was detached from the external oblique muscle, and (D) size was measured as $10 \times 9.5 \times 5.5 \mathrm{~cm}$.

Ethicon, Somerville, NJ, USA). A strong traction force was not required to secure the operative field of view in this procedure (Fig. 3B-D). The entire operation took 40 minutes. The ultrasonic harmonic scalpel simultaneously accomplished hemostasis and dissection, and although the operation time was reduced, the dissection was performed meticulously. No perioperative complications were noted. Postoperatively, the patient was esthetically satisfied with the 3.5-cm-long scar (Figs. 4, 5).

\section{DISCUSSION}

Lipoma is a benign tumor originating from the mesenchyme, and it is the most frequent type of soft tissue tumor [1]. Giant intramuscular lipomas are deep-seated lipomas that measure greater than $10 \mathrm{~cm}$ in any dimension [3], and an extended incision length may be necessary to achieve complete resection of the lipoma and its entire capsule. In this report, an ultrasonic harmonic scalpel was used to ensure full visualization of the operation field and easily remove a $10 \times 10-\mathrm{cm}$ giant intramuscular lipoma with a minimal in- 


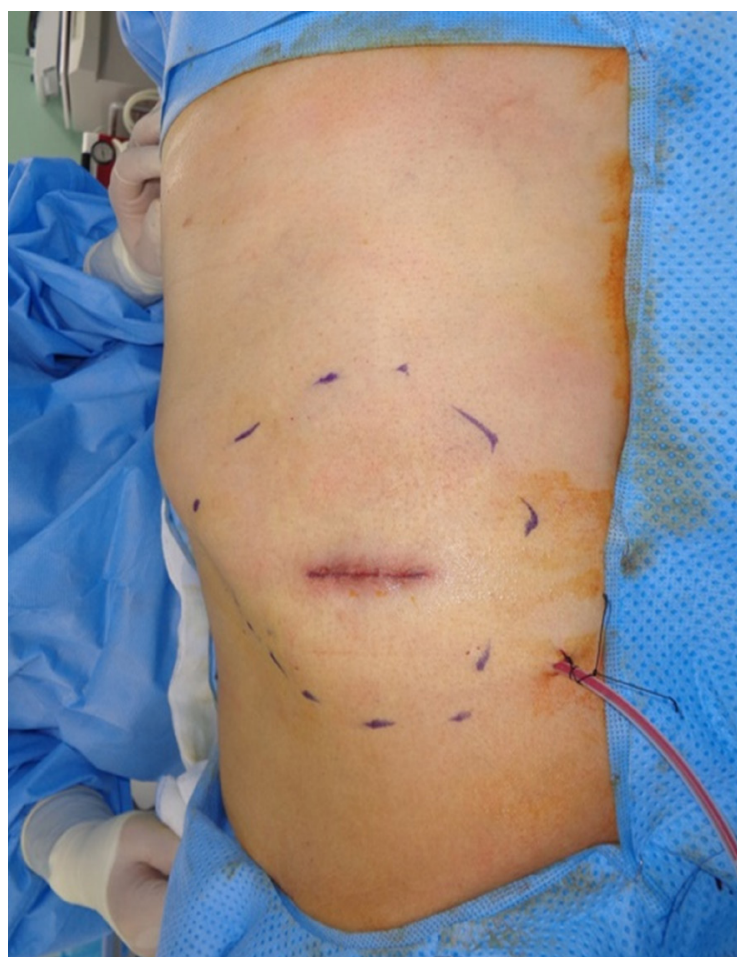

Fig. 4. An immediate postoperative clinical photo showing a 3.5-cmlong wound without skin damage.

cision length.

In the traditional excision procedure, utilizing a small incision to shorten the postoperative scar requires a high traction force to obtain the appropriate visual field; as a result, tissue and incision margin injury might occur, leading to poor wound healing or hypertrophic scar formation [6,7].

In contrast, the ultrasonic harmonic scalpel is a surgical instrument frequently used in laparoscopic surgery that provides an appropriate field of view for the operation with small incisions and without the requirement of a strong force of traction. Minimizing mechanical forces in the wound environment improves wound healing and reduces scar formation [6].

The ultrasonic harmonic scalpel can simultaneously perform cutting and cauterization. Thus, it can be used to perform a meticulous operation and to shorten the operation time. Moreover, the lateral thermal spread of the harmonic scalpel is minimal, so it may be helpful for minimizing thermal damage around the tissue [12].

Moreover, using the harmonic scalpel reduces the occurrence of seroma, because the ultrasound energy of the device prevents the opening through which serous discharge occurs. However, dissection with this energy-delivering device in a narrow field is predicated on sufficient anatomical knowledge. By ensuring a thorough understanding of the blood vessels and nerves to be dissected in the region of the lipoma, intraoperative bleeding can be minimized and the sequelae after surgery can be reduced.

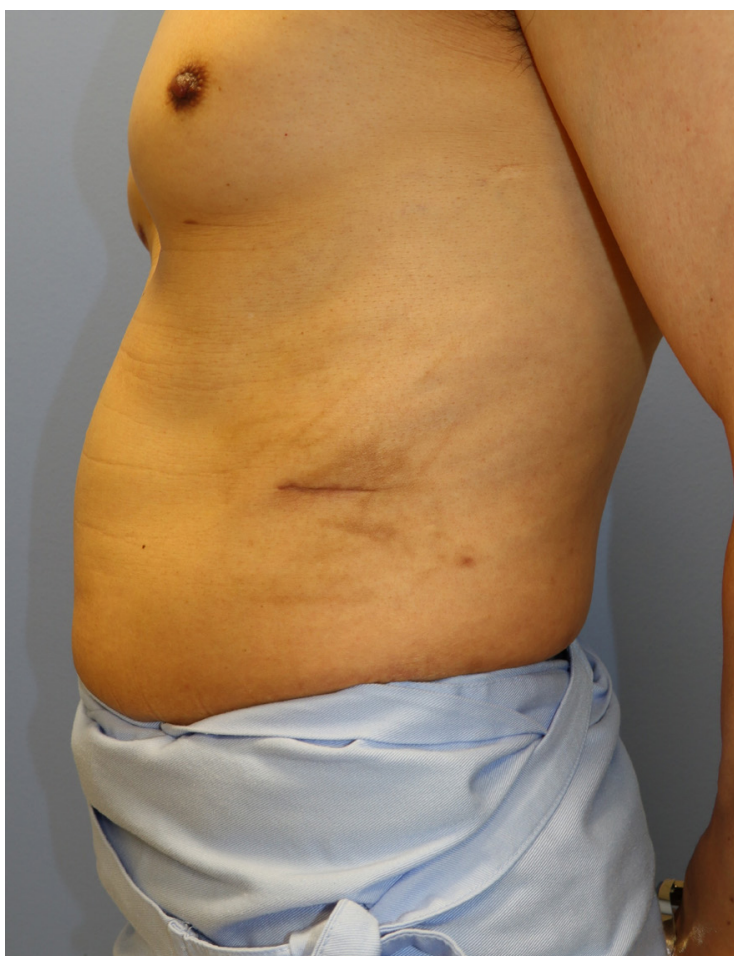

Fig. 5. At 3 months postoperatively, a 3.5-cm-long scar was present without hypertrophy.

Various surgical techniques that improve esthetic outcomes by shortening scar length have been reported, including suction-assisted surgical excision, endoscopic-assisted liposuction, and endoscopic excision for removing moderate and large lipomas [8-10]. Liposuction is one of the most helpful options for removing large, diffuse-spreading, and ill-defined suprafascial lipomas [13]. The advantages of liposuction include smaller scars, less pain, cost-effectiveness, reduced operative time, lower complication rates, and a better final contour [11]. However, the lipoma tissue is fragmented in the process of liposuction, and it is difficult to perform an accurate pathological analysis. Therefore, a preoperative fine needle aspiration biopsy or magnetic resonance imaging should be considered in patients with a questionable mass [14]. Moreover, a higher recurrence risk due to tissue fragmentation has been reported $[11,15]$.

Compared with the previously reported methods, the ultrasonic harmonic scalpel has a lower recurrence rate and has the advantage of permitting lipoma excision en bloc, even in large and deep-seated lipomas, so that pathologic specimens can be precisely analyzed.

\section{NOTES}

\section{Conflict of interest}

No potential conflict of interest relevant to this article was reported. 


\section{Ethical approval}

The study was performed in accordance with the principles of the Declaration of Helsinki.

\section{Patient consent}

The patient provided written informed consent for the publication and the use of his images.

\section{ORCID}

Jun Hyeok Kim

Yeon Ji Lee

Deuk Young Oh https://orcid.org/0000-0003-4657-2090

https://orcid.org/0000-0002-8298-4036

https://orcid.org/0000-0003-3499-1554

\section{REFERENCES}

1. Murphey MD, Carroll JF, Flemming DJ, et al. From the archives of the AFIP: benign musculoskeletal lipomatous lesions. Radiographics 2004; 24:1433-66.

2. McTighe S, Chernev I. Intramuscular lipoma: a review of the literature. Orthop Rev (Pavia) 2014;6:5618.

3. Sanchez MR, Golomb FM, Moy JA, et al. Giant lipoma: case report and review of the literature. J Am Acad Dermatol 1993;28(2 Pt 1):2668.

4. Warner JJ, Madsen N, Gerber C. Intramuscular lipoma of the deltoid causing shoulder pain: report of two cases. Clin Orthop Relat Res 1990; (253):110-2.

5. Gutknecht DR. Painful intramuscular lipoma of the thigh. South Med
J 2004;97:1121-2

6. Barnes LA, Marshall CD, Leavitt T, et al. Mechanical forces in cutaneous wound healing: emerging therapies to minimize scar formation. Adv Wound Care (New Rochelle) 2018;7:47-56.

7. Bush JA, McGrouther DA, Young VL, et al. Recommendations on clinical proof of efficacy for potential scar prevention and reduction therapies. Wound Repair Regen 2011;19 Suppl 1:s32-7.

8. Sakai Y, Okazaki M, Kobayashi S, et al. Endoscopic excision of large capsulated lipomas. Br J Plast Surg 1996;49:228-32.

9. Al-basti HA, El-Khatib HA. The use of suction-assisted surgical extraction of moderate and large lipomas: long-term follow-up. Aesthetic Plast Surg 2002;26:114-7.

10. Sawaizumi M, Maruyama Y, Onishi K, et al. Endoscopic extraction of lipomas using an ultrasonic suction scalpel. Ann Plast Surg 1996;36: 124-8.

11. Coleman WP 3rd. Noncosmetic applications of liposuction. J Dermatol Surg Oncol 1988;14:1085-90.

12. Choi CW, Kim BJ, Moon SE, et al. Treatment of lipomas assisted with tumescent liposuction. J Eur Acad Dermatol Venereol 2007;21:243-6.

13. Peev I, Spasevska L, Mirchevska E, et al. Liposuction assisted lipoma removal - option or alternative? Open Access Maced J Med Sci 2017;5: 766-70.

14. Dalal KM, Antonescu CR, Singer S. Diagnosis and management of lipomatous tumors. J Surg Oncol 2008;97:298-313.

15. Wilhelmi BJ, Blackwell SJ, Mancoll JS, et al. Another indication for liposuction: small facial lipomas. Plast Reconstr Surg 1999;103:1864-7. 